\title{
Real Time 100 Gbit/s Electrical Nyquist WDM Transmitter
}

\author{
A. D. Ellis, M. E. McCarthy, D. Lavery*, N. Mac Suibhne, S. Sygletos, S. Savory*, B.Thomsen*, \\ R. Killey*, P. Bayvel* and P. Harper \\ Aston Institute of Photonic Technology, Aston University, James Watt Queensway, Birmingham B4 7ET, UK \\ * Optical Networks Group, University College London, Torrington Place, London, WC1E 7JE, UK \\ Tel: +44 121204 3075, e-mail: andrew.ellis@aston.ac.uk
}

\begin{abstract}
We demonstrate the use of passive electrical filters to produce high quality spectrally-shaped 29Gbaud WDM signals with a $20 \mathrm{~dB}$ bandwidth of $32 \mathrm{GHz}$, a roll-off of $4.8 \mathrm{~dB} / \mathrm{GHz}$ and a required OSNR of $12.1 \mathrm{~dB}$.
\end{abstract}

Keywords: Wavelength division multiplexing. Modulation Formats

\section{INTRODUCTION}

Optical networks currently face two key challenges, capacity [1] and energy consumption [2]. Since the introduction of optical super-channels [3], the adoption of coherent detection has enabled proposals for superchannels based on all-optical OFDM [4], Nyquist-WDM [5] and FFT based OFDM [6]. In addition to an increase in information spectral density (ISD), such techniques also enable flex-grid optical networks offering an additional throughput of around 30\% [7]. Of these, Nyquist-WDM is currently receiving considerable attention because it simultaneously offers both a near- rectangular spectrum and a moderate peak-to-average power ratio. Competing formats suffer from high peak-to-average power ratio (FFT-based OFDM) or spectral roll-off (alloptical OFDM). However, whilst early studies of Nyquist-WDM employed optical filters [8] additional digital signal processing is typically required at the transmitter in order to achieve the benefits of enhanced ISD without inducing penalties in the required signal-to-noise ratio, a benefit which is shared with all-optical OFDM [9]. Recent results [10] have suggested that the impulse response (or frequency resolution) of the shaping filter is critical, demonstrating a clear advantage $(\sim 0.8 \mathrm{~dB})$ for Nyquist filtering in the digital domain using 2048 taps when compared to state-of-the-art optical (12 GHz bandwidth) filters and off-the-shelf electrical filters for a symbol rate of 20 Gbaud. Direct electrical filtering is also used in frequency and time-packed transmission systems, but at the expense of requiring multi-symbol detection schemes which compensate for intended intersymbol interference and filter imperfections [11].

In this paper, we demonstrate Nyquist signal shaping in excess of 28 Gbaud using a cascade of two off-the-shelf analog electrical filters and conventional digital modulator drivers. To the best of our knowledge this represents the highest symbol rate for electronically- generated Nyquist WDM. A required OSNR (at a BER of 10-3) is achieved for a polarization multiplexed (PM) $100 \mathrm{Gbit} / \mathrm{s}$ system, with an additional power consumption of only $400 \mathrm{~mW}(140 \mathrm{~mA}$ at $3.3 \mathrm{~V})$ for the 9-tap finite impulse response filter suggesting that this is simultaneously the lowest power consumption electronically generated Nyquist WDM technique reported to date. These results demonstrate that the power consumption associated with transmitter side digital signal processing may be effectively neglected using simple analog filters without any significant performance penalty, even using simple symbol-by-symbol detection.

\section{SYSTEM CONFIGURATION}
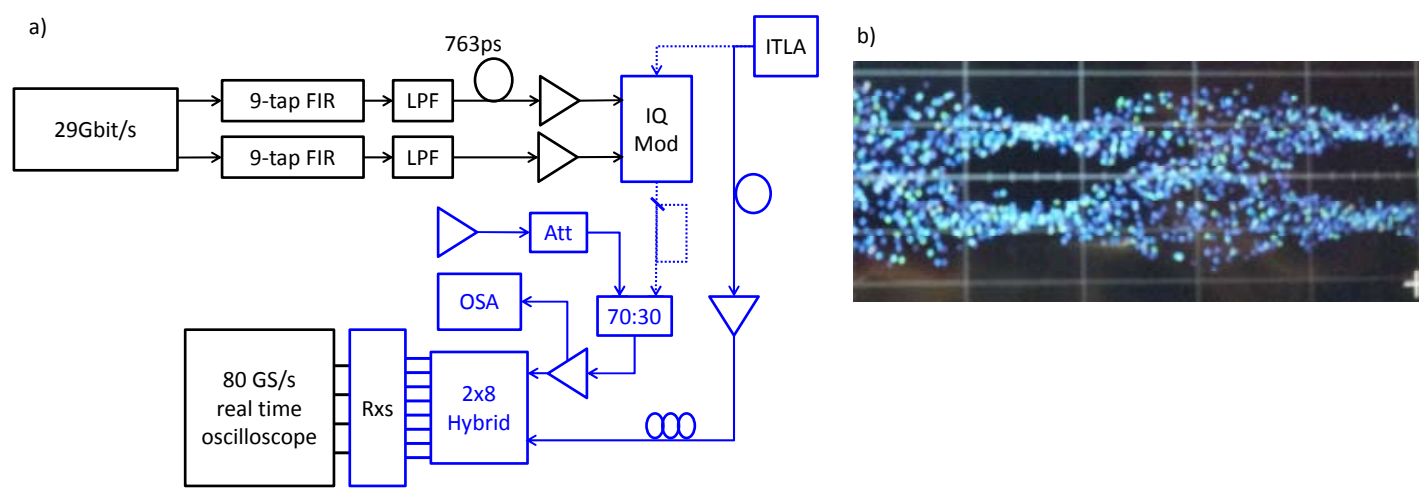

Figure 1: a) Schematic diagram of electrical generation of Nyquist signals and their coherent detection. PPGpulse pattern generator, LPF-low pass filter, ITLA-tunable laser (100kHz linewidth), Att: 100GHz filter plus attenuator, OSA-optical spectrum analyser. Electrical connections are shown in black and optical fibres in blue (dashed lines indicating polarization maintaining fibres).b) Eye diagram of pre-filtered drive signal. 
Analog electrical filters may be readily fabricated with almost arbitrary roll-off factors [11], however they are typically accompanied by sharp variations in group delay near the filter edge, and ripple within the pass-band. Here, we employ a commercially available low pass filter with a 14.6 GHz bandwidth, (Marki FLP-1460) accompanied by an adaptive 9-tap finite impulse response (FIR) filter originally developed for electronic dispersion compensation of directly detected 32 Gbaud signals (Hittite HMC6545LP5E) for application in the cost and power sensitive $100 \mathrm{GbE}$, Infiniband and Fibre Channel [12] markets. The filter had a specified tap spacing of approximately 18 ps, a net latency of less than 200 ps and an output rise time of approximately 16 ps. As shown in Figure 1, the combined filters were placed at the output of a pulse pattern generator generating a 29 Gbaud, 215-1 pseudo random bit sequence, with the tap coefficients initially set to the Fourier transform of the desired filter response, and subsequently optimized to minimize the electrical receiver sensitivity when the filter output was connected to an error detector, whilst maintaining a rectangular RF spectrum. This was necessary to compensate for the individual frequency response of the tap paths. The eye diagram at this point is shown in Figure 1b, whilst the spectrum, measured using an $80 \mathrm{Gs} / \mathrm{s}$ real time oscilloscope is shown in Figure 2.

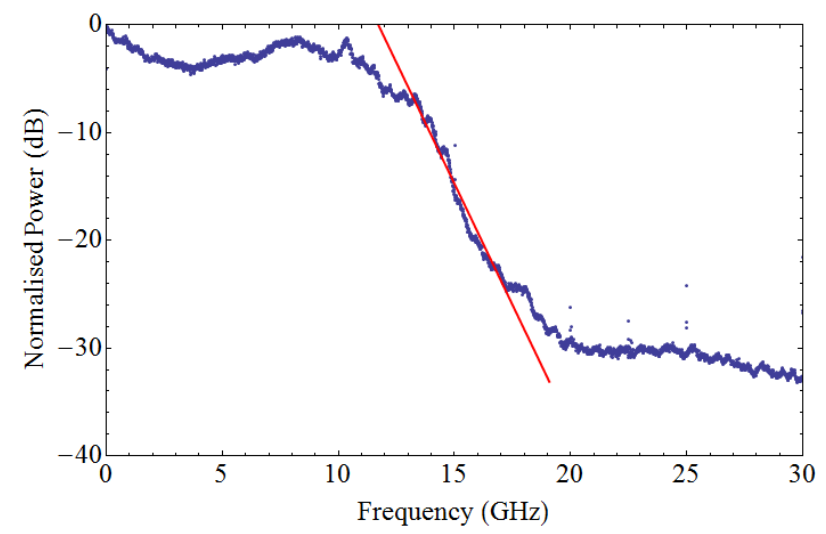

Figure 2: Electrical spectrum of electronically generated Nyquist WDM drive signal (blue) and fit to edge of signal spectrum (red) showing $4.8 \mathrm{~dB} / \mathrm{GHz}$ roll off.

The signals were amplified with drive amplifiers optimized for conventional digital signals, and used to drive a Lithium Niobate dual parallel Mach-Zehnder modulator with data and delayed (22 bit periods) inverse data signals. Different filter paths were used for each drive signal. The spectrum of the resultant single polarization QPSK signal is shown in Figure 3 for both low (red) and high (green) peak to peak drive signals showing a slope of $4.8 \mathrm{~dB} / \mathrm{GHz}$ (20 dB in less than $15 \%$ of the symbol rate). The signal was polarization multiplexed using a delay and add configuration and combined with amplified spontaneous emission noise, filtered to a bandwidth of $100 \mathrm{GHz}$ and centred on the signal. The combined signal and noise was further amplified to $20 \mathrm{dBm}$, where the optical signal to noise ratio was monitored and combined with a delayed copy of the transmitter laser (also amplified to $20 \mathrm{dBm}$ ) in a 90 degree optical hybrid (2x8) for coherent detection. The signals were detected using four $50 \mathrm{GHz}$ bandwidth balanced photodiodes and digitized at $80 \mathrm{GS} / \mathrm{s}$ using a real time oscilloscope with a 25 $\mathrm{GHz}$ analog bandwidth.

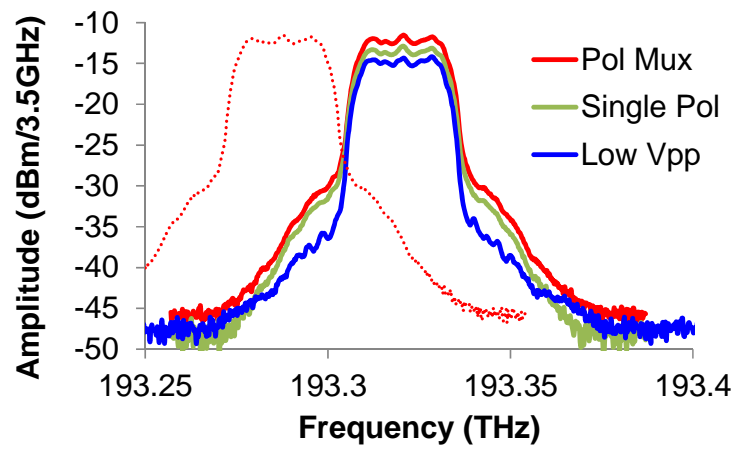

Figure 3: Optical spectra of pre-filtered QPSK signals for a low drive amplitude (blue), a drive amplitude optimized for best BER performance (green) and after polarization multiplexing (red). Also shown is a further copy of the polarization multiplexed signal, shifted by $33 \mathrm{GHz}$ to illustrate the potential WDM capability of the transmitter.

The coherently-detected signal was processed off-line using a conventional DSP algorithm developed for unfiltered PM-QPSK signals. The adaptive equaliser used a 15-tap (T/2 spaced) filter, optimised using a constant 
modulus algorithm, Viterbi and Viterbi phase recovery using a window length of 64 samples. Hard decisions were made on the QPSK signal followed by bit error ratio (BER) calculations. No digital filtering was employed to enhance the signal to noise ratio. We anticipate an additional penalty of up to $1 \mathrm{~dB}$ from the excess beat noise associated with omitting this filter. We intially observed that the signal was degraded by a certain level of variable transmitter phase bias (no stabilisation ciruit was employed). To accommodate this, we adjusted the equaliser configuration following the work of [12]. This resulted in an improvement of the required OSNR penalty of around $0.7 \mathrm{~dB}$ in the BER range $\left\{10^{-2}\right.$ to $\left.10^{-3}\right\}$ and a considerable reduction in the variability of each BER measurement. It is clear that employing transmitter phase bias stabilisation would improve the results further.

\section{RESULTS}

Typical received constellations after signal processing are shown in Figure 4 for a polarization multiplexed QPSK signal including the shaping filter. A small level of residual distortion of the Gaussian noise clouds is apparent, suggesting that there is a small level of residual inter-symbol interference This is confirmed by the finite thickness of the drive signal eye diagram (Figure 1b).

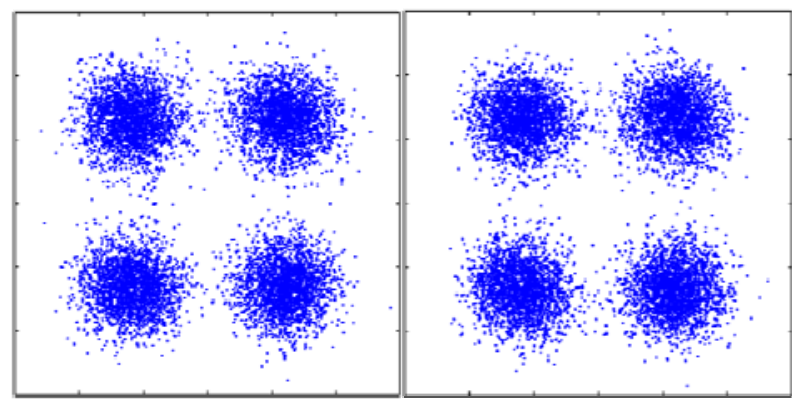

Figure 4: Typical received constellation diagrams for a Nyquist filtered polarization multiplexed QPSK signal, obtained with an OSNR of $16.4 \mathrm{~dB}$ and a corresponding BER of $1.510^{-3}$. Left: X-polarisation and Right: Ypolarisation.

Measured bit error rate characteristics are shown in Figure 5 for PM-QPSK signals. The required OSNR for a bit error rate of $10^{-3}$ is $15.7 \mathrm{~dB}$ giving a net throughput of $107 \mathrm{Gbit} / \mathrm{s}$ (assuming 7\% overhead), whilst for a bit error rate of $10^{-2}$ (96 Gbit/s assuming 20\% overhead) the required OSNR is $12.1 \mathrm{~dB}$. These results are comparable to recently reported results using digitally implemented 2048-tap filters [10] after correction for the higher symbol rate reported here. Note that receiver side matched filtering was not implemented in this work (other than through the 15-Tap equalizer) and so, we observe a small power penalty (less than $1.3 \mathrm{~dB}$ ) and, at lower bit error rates, an error floor. The possibility of designing a digital matched filter for this filter profile is under investigation.

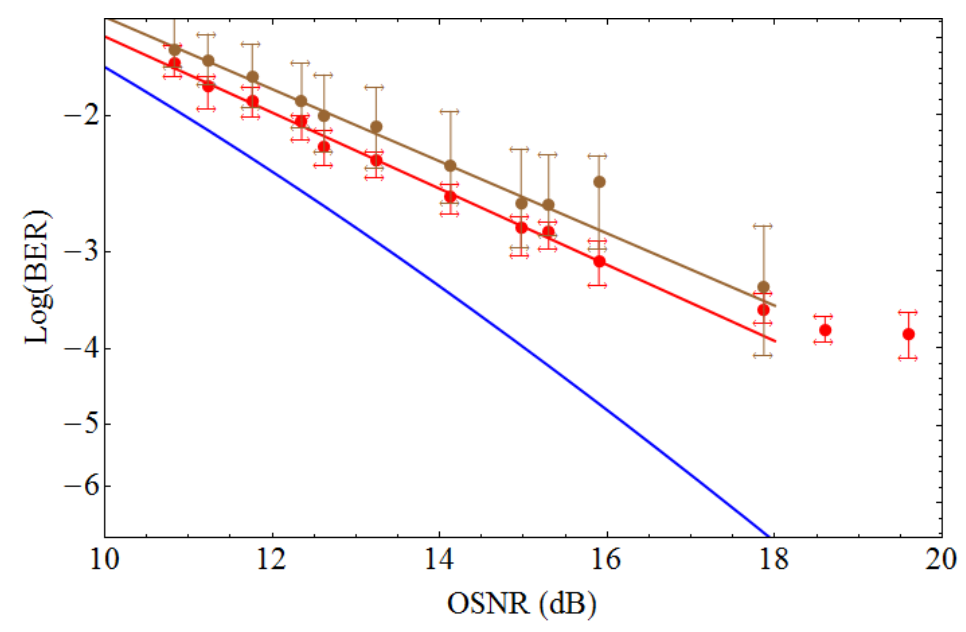

Figure 5: Bit error rate characterization of 29 Gbaud QPSK signals showing polarization multiplexed electrically filtered QPSK with conventional (brown) or optimised [12] (red) equaliser with a linear curve fits included as a guide to the eye and theortical limit for PM-QPSK (blue). Data points represent the mean BER from 10 data sets each of 80 ksamples, whilst error bars represent best and worst data sets at each OSNR. 


\section{CONCLUSIONS}

In this paper we have demonstrated a simple analog filter based technique to generate PM-QPSK signals with quasi rectangular spectra (roll-off of $4.8 \mathrm{~dB} / \mathrm{GHz}$ ). The resultant 29 Gbaud signals show negligible OSNR penalty when compared to other forms of Nyquist signal generation. This represents the highest symbol rate for electronically generated Nyquist WDM reported to date. With an additional power consumption of less than 400 $\mathrm{mW}$, we anticipate that the use of such simple adaptive analog filters will enable the generation of high capacity Nyquist-WDM signals in a power efficient manner enabling the capacity of communication systems to be increased without significant increases in the energy consumption per bit.

\section{ACKNOWLEDGEMENTS}

This work was partly funded by the European Communities 7th Framework Programme (Grant 318415-FOX-C), EPSRC (Grant EP/J017582/1-UNLOC), and The Royal Society (Grant WM120035-TEST). The authors would like to thank Dr I. Philips and Mr R.Able for development of the coherent testbed used in this experiment and Dr D. Hillerkuss (ETH Zurich) for useful discussions.

\section{REFERENCES}

[1] R. J. Essiambre, G. Kramer, P. J. Winzer, G. J. Foschini, and B. Goebel, "Capacity limits of optical fiber networks," J. Lightwave Technol. 28 (4), 662-701, (2010).

[2] R. S. Tucker, "Green Optical Communications-Part I: Energy Limitations in Transport," IEEE Journal of Selected Topics in Quantum Electronics 17, 245-260 (2011).

[3] A.D.Ellis, and F.C.G.Gunning, "Spectral Density Enhancement using Coherent WDM", Photonics Technology Letters, 17, (2), 504-506, (2005).

[4] S. Chandrasekhar, X. Liu; B. Zhu, and D.W. Peckham, "Transmission of a 1.2-Tb/s 24-carrier no-guardinterval coherent OFDM superchannel over 7200-km of ultra-large-area fiber," European Conference on Optical Communication, (2009).

[5] D. Hillerkuss, et al., "Single-laser 32.5 Tbit/s Nyquist WDM transmission," Journal of Optical Communications and Networking, 4,(10), 715,723, (2012).

[6] Y. Ma, Q. Yang, Y. Tang, S. Chen, and W. Shieh, "1-Tb/s single-channel coherent optical OFDM transmission over 600-km SSMF fiber with subwavelength bandwidth access," Opt. Express 17, 94219427 (2009).

[7] L. Velasco, P. Wright, A. Lord, and G. Junyent, "Saving CAPEX by Extending Flexgrid-based Core Optical Networks toward the Edges [Invited]," J. Opt. Commun. Netw. 5, A171-A183 (2013).

[8] G. Bosco, A. Carena, V. Curri, P. Poggiolini, E. Torrengo,and F, Forghieri,, "Investigation on the robustness of a Nyquist-WDM Terabit superchannel to transmitter and receiver non-idealities," European Conference and Exhibition on Optical Communication (ECOC), (2010).

[9] J. Zhao and A. D. Ellis, "Electronic Impairment Mitigation in Optically Multiplexed Multicarrier Systems”, Journal of Lightwave Technology, 29, (3), 278, (2011).

[10] R. Schmogrow, S. Ben-Ezra, P. C. Schindler, B. Nebendahl, C. Koos, W. Freude, and J. Leuthold, "PulseShaping With Digital, Electrical, and Optical Filters-A Comparison,” IEEE J. Lightwave Technol., 31, (15), 2570-2577 (2013).

[11] F. Fresi, M. Secondi, G. Berrettini, G. Meloni, and L. Poti, "Impact of optical and electrical narrowband spectral shaping in faster than Nyquist Tb superchannel,” IEEE Photon. Tech. Lett 25, 2301-2303 (2013).

[12] B. McGreevy, "Hittite Launches 32 Gbps, dual channel advanced linear equaliser" downloaded March 2014 from www.hittite.com/press_releases/index.html/view/723, (2013).

[13] M. Paskov, D. Lavery, and S.J. Savory, "Blind Equalization of Receiver In-Phase/Quadrature Skew in the Presence of Nyquist Filtering," Photonics Technology Letters, 25, 2446-2449, (2013). 